\title{
Coupling of Nonlocal Potentials to Electromagnetic Fields
}

\author{
Sohrab Ismail-Beigi, Eric K. Chang, and Steven G. Louie \\ Department of Physics, University of California, Berkeley, CA 94720, and \\ Materials Sciences Division, Lawrence Berkeley National Laboratory, Berkeley, CA 94720
}

October 27, 2018

\begin{abstract}
Nonlocal Hamiltonians are used widely in first-principles quantum calculations; the nonlocality stems from eliminating undesired degrees of freedom, e.g. core electrons. To date, attempts to couple nonlocal systems to external electromagnetic (EM) fields have been heuristic or limited to weak or long wavelength fields. Using Feynman path integrals, we derive an exact, closed-form coupling of arbitrary EM fields to nonlocal systems. Our results justify and clarify the couplings used to date and are essential for systematic computation of linear and especially nonlinear response.
\end{abstract}

Theoretical study of the electronic response of quantum systems to electromagnetic (EM) probes is central to understanding experimental results and what they imply about the structure of matter. It is now possible to predict the response, from first principles, with sufficient accuracy to compare quantitatively to experiment [1]. Nonlocal pseudopotentials are a key ingredient of many ab initio electronic structure calculations as they eliminate the largely inert and thus physically unimportant core electrons. The price paid is that the valence electrons feel a nonlocal atomic potential.

Hence, it is vital to know how electrons moving in nonlocal potentials couple to EM fields. Early work [2] found the coupling within the effective-mass framework. To date, refs. [3, [4, 5] represent the only first principles attempts to find the coupling, but with limited success. In ref. [3], the coupling terms involve an integral of the field over an unspecified path. Path ambiguity, discussed below, is a central problem that leads to non unique couplings and incorrect quantitative results. Ref. [4] stops at the formal level due to operator ordering ambiguities, and an explicit, practical form of the coupling is given only for the long wavelength (i.e. dipole) approximation. This treatment excludes the description of AC magnetic fields or, more generally, spatially varying EM fields. Operator ordering problems also force the results of ref. [5] to be limited to linear (i.e. weak) coupling.

Other attempts are also limited to linear coupling [6, 7] where physical intuition leads to replacing the momentum by the velocity operator. When seeking nonlinear response, the coupling is either considered only for longitudinal fields [8, 9] or only within the long wavelength (dipole) approximation [10].

Our work presents a rigorous derivation of an explicit, closed-form expression for the coupling of nonlocal systems to arbitrary EM fields. The value of this result is several fold: e.g., having the correct coupling is essential for accurate and systematic $a b$ initio calculation of linear and nonlinear response. Aside from first principles methods, many semi-empirical or phenomenological models use nonlocal Hamiltonians with tight-binding like hopping terms. There have been only heuristic attempts to couple such systems to EM fields [11]. Our results justify the assumed forms of previous couplings.

Although ab initio calculations involve interacting electrons, nonlocal potentials are one-body operators. For clarity, we derive our results for a one-electron system. The results generalize directly to the interacting case.

A main principle of electrodynamics is gauge-invariance. A particle in a local potential $U(\mathbf{r})$ is governed by the Hamiltonian $\hat{H}_{0}(\hat{\mathbf{r}}, \hat{\mathbf{p}})=\hat{\mathbf{p}}^{2} / 2 m+\hat{U}(\hat{\mathbf{r}})$. The standard prescription for the gauge-invariant coupling uses the minimal substitution $\hat{\mathbf{p}} \rightarrow \hat{\mathbf{\Pi}} \equiv \hat{\mathbf{p}}-\hat{\mathbf{A}}(\hat{\mathbf{r}}, t)$ (A is the vector potential). The coupled Hamiltonian is $\hat{H}_{\mathbf{A}}(\hat{\mathbf{r}}, \hat{\mathbf{p}})=\hat{\mathbf{\Pi}}^{2} / 2 m+\hat{U}(\hat{\mathbf{r}})+q \hat{\phi}(\hat{\mathbf{r}}, t)(\phi$ is the electrostatic potential). We use units where $q / c=\hbar=1(q$ is the particle's charge and $c$ the speed of light). 
The problem of interest, however, has a nonlocal potential $V_{n l}$ with Hamiltonian

$$
\hat{H}_{0}=\hat{\mathbf{p}}^{2} / 2 m+\hat{U}(\hat{\mathbf{r}})+\hat{V}_{n l},
$$

where $V_{n l}\left(\mathbf{r}, \mathbf{r}^{\prime}\right) \equiv\left\langle\mathbf{r}\left|\hat{V}_{n l}\right| \mathbf{r}^{\prime}\right\rangle \neq 0$ for $\mathbf{r} \neq \mathbf{r}^{\prime}$. Gauge-invariance demands that $V_{n l}$ depend on A. A major obstacle to finding $\hat{H}_{\mathbf{A}}$ is that $V_{n l}\left(\mathbf{r}, \mathbf{r}^{\prime}\right)$ is not specified in terms of the canonical operators $(\hat{\mathbf{r}}, \hat{\mathbf{p}})$. Below, we first rewrite $V_{n l}$ in terms of $(\hat{\mathbf{r}}, \hat{\mathbf{p}})$, and then perform minimal substitution in an unambiguous manner to find $\hat{H}_{\mathbf{A}}$.

Starting with $\hat{V}_{n l}$, we perform a Weyl transformation to obtain an equivalent operator $\hat{W}$ of $(\hat{\mathbf{r}}, \hat{\mathbf{p}})$ [12]:

$$
\hat{W}(\hat{\mathbf{r}}, \hat{\mathbf{p}})=\int d \mathbf{s} e^{-i \hat{\mathbf{p}} \cdot \mathbf{s} / 2} V_{n l}\left(\hat{\mathbf{r}}+\frac{\mathbf{s}}{2}, \hat{\mathbf{r}}-\frac{\mathbf{s}}{2}\right) e^{-i \hat{\mathbf{p}} \cdot \mathbf{s} / 2} .
$$

It is straightforward to show that $\left\langle\mathbf{r}|\hat{W}(\hat{\mathbf{r}}, \hat{\mathbf{p}})| \mathbf{r}^{\prime}\right\rangle=\left\langle\mathbf{r}\left|\hat{V}_{n l}\right| \mathbf{r}^{\prime}\right\rangle$. Thus $\hat{V}_{n l}$ and $\hat{W}$ are the same operator. We now have written the Hamiltonian without fields as

$$
\hat{H}_{0}(\hat{\mathbf{r}}, \hat{\mathbf{p}})=\hat{\mathbf{p}}^{2} / 2 m+\hat{U}(\hat{\mathbf{r}})+\hat{W}(\hat{\mathbf{r}}, \hat{\mathbf{p}}) .
$$

For the equivalent classical system, $\mathcal{W}(\mathbf{r}, \mathbf{p})$ is a function of the numbers $\mathbf{r}$ and $\mathbf{p}$ given by

$$
\mathcal{W}(\mathbf{r}, \mathbf{p})=\int d \mathbf{s} V_{n l}(\mathbf{r}+\mathbf{s} / 2, \mathbf{r}-\mathbf{s} / 2) e^{-i \mathbf{p} \cdot \mathbf{s}} .
$$

At this stage, we would like to employ the minimal substitution $\hat{\mathbf{p}} \rightarrow \hat{\boldsymbol{\Pi}}$ in Eq. (3) to find the coupled Hamiltonian $\hat{H}_{\mathbf{A}}$. Unfortunately, the components of $\hat{\boldsymbol{\Pi}}$ do not commute so that initially equivalent orderings of operators in $\hat{W}$ lead to physically different couplings: e.g., $\hat{\mathbf{p}}_{x} \hat{\mathbf{p}}_{y}=\hat{\mathbf{p}}_{y} \hat{\mathbf{p}}_{x}$ but $\hat{\boldsymbol{\Pi}}_{x} \hat{\boldsymbol{\Pi}}_{y} \neq \hat{\boldsymbol{\Pi}}_{y} \hat{\boldsymbol{\Pi}}_{x}$ since $\left[\hat{\mathbf{\Pi}}_{x}, \hat{\mathbf{\Pi}}_{y}\right]=i \mathbf{B}_{z}$. Continuing along this line requires applying ordering postulates to the operators in $\hat{W}$ before minimal substitution to guarantee unambiguous final results. Only for long wavelengths (i.e. spatially uniform $\mathbf{A}$ ) is the substitution unambiguous, and the results of [4, 10] are limited to this case. Similarly, this same ordering issue forced the results in [5] be limited to first order in $\mathbf{A}$.

An equivalent statement of the problem is that different evaluations of $e^{-i \hat{\mathbf{p}} \cdot \mathbf{s} / 2}$ in Eq. (2) correspond to translating by $\mathbf{s} / 2$ along different paths. This path ambiguity is present in [3] where line integrals of $\mathbf{A}$ are used. When $\mathbf{A}$ is constant, all paths give identical results. But once $\mathbf{A}$ has spatial variation, different paths contribute different phases leading to non unique differing couplings.

To find the solution, we first consider the classical system governed by $\mathcal{W}(\mathbf{r}, \mathbf{p})$. Minimal substitution is well defined classically since $\mathbf{p}$ and $\mathbf{r}$ are commuting numbers. The classical coupled Hamiltonian is

$$
\mathcal{H}_{\mathbf{A}}(\mathbf{r}, \mathbf{p})=\boldsymbol{\Pi}^{2} / 2 m+U(\mathbf{r})+\mathcal{W}(\mathbf{r}, \boldsymbol{\Pi})+\phi(\mathbf{r}, t) .
$$

Next, the Feynman path-integral formulation provides the quantum evolution of a system by summing over its classical dynamics. The propagation amplitude $K$ between the two space-time points $\left(\mathbf{r}^{\prime}, 0\right)$ and $(\mathbf{r}, t)$ in the operator formulation is given by [13]

$$
\begin{aligned}
K\left(\mathbf{r}, t ; \mathbf{r}^{\prime}, 0\right) & \equiv\left\langle\mathbf{r}\left|T \exp \left(-i \int_{0}^{t} d \tau \hat{H}_{\mathbf{A}}(\tau)\right)\right| \mathbf{r}^{\prime}\right\rangle \\
& =\delta\left(\mathbf{r}-\mathbf{r}^{\prime}\right)-i t\left\langle\mathbf{r}\left|\hat{H}_{\mathbf{A}}(t)\right| \mathbf{r}^{\prime}\right\rangle+O\left(t^{2}\right)
\end{aligned}
$$

Now $K$ also equals the result of the Feynman formulation where we sum over all classical trajectories in phase space $(\mathbf{r}, \mathbf{p})$ weighted by each path's action $S$ [14]:

$$
\begin{aligned}
K\left(\mathbf{r}, t ; \mathbf{r}^{\prime}, 0\right) & =\int^{\prime} D[\mathbf{r}] \int D[\mathbf{p}] e^{i S} \\
S & =\int_{0}^{t} d \tau\left[\mathbf{p}(\tau) \cdot \dot{\mathbf{r}}(\tau)-\mathcal{H}_{\mathbf{A}}(\mathbf{r}(\tau), \mathbf{p}(\tau))\right]
\end{aligned}
$$

The prime on the $\mathbf{r}$ path integral means that we only sum over paths where $\mathbf{r}(0)=\mathbf{r}^{\prime}$ and $\mathbf{r}(t)=\mathbf{r}$. 
We seek the quantum Hamiltonian $\hat{H}_{\mathbf{A}}(t)$ which is the $O(t)$ term in Eq. (6). Hence we will expand the path integral of Eq. (7) to $O(t)$ and extract $\hat{H}_{\mathbf{A}}(t)$.

We now use the fact that $\mathcal{W}(\mathbf{r}, \mathbf{p})$ is generally bounded, i.e. $|\mathcal{W}(\mathbf{r}, \mathbf{p})|<C$ for some number $C$. This is true for atomic pseudopotentials $V_{n l}\left(\mathbf{r}, \mathbf{r}^{\prime}\right)$ which are smooth and have compact support. Thus when $t \rightarrow 0$, the contribution of the $\mathcal{W}$ term to $S$ in Eq. (8) is $O(t)$. Hence we need expand only to linear order in $\mathcal{W}$ :

$$
\begin{aligned}
K & =K_{l o c}+K_{n l}, \\
K_{l o c} & =\int^{\prime} D[\mathbf{r}] \int D[\mathbf{p}] e^{i S_{l o c}}, \\
K_{n l} & =-i \int^{\prime} D[\mathbf{r}] \int D[\mathbf{p}] \int d \tau \mathcal{W}(\mathbf{r}, \mathbf{\Pi}) e^{i S_{l o c}}, \\
S_{l o c} & =\int d \tau^{\prime}\left[\mathbf{p} \cdot \dot{\mathbf{r}}-\Pi^{2} / 2 m-U(\mathbf{r})-\phi\left(\mathbf{r}, \tau^{\prime}\right)\right] .
\end{aligned}
$$

The local propagator $K_{l o c}$ describes the textbook situation of a local potential and is given by

$$
K_{l o c}=\delta\left(\mathbf{r}-\mathbf{r}^{\prime}\right)-i t\left\langle\mathbf{r}\left|\hat{H}_{l o c}\right| \mathbf{r}^{\prime}\right\rangle+O\left(t^{2}\right),
$$

where $\hat{H}_{l o c}=\hat{\mathbf{\Pi}}^{2} / 2 m+\hat{U}+q \hat{\phi}$. Thus, we concentrate on the nonlocal propagator $K_{n l}$.

The local potential $U+q \phi$ depends only on $\mathbf{r}(\tau)$ and $\tau$ and not on p. Hence as $t \rightarrow 0$, the contribution to $S_{l o c}$ from $U+q \phi$ is $O(t)$. We ignore this contribution to $K_{n l}$ since it leads to terms of $O\left(t^{2}\right)$. However, $p$ scales as $\mathbf{p} \sim\left(\mathbf{r}-\mathbf{r}^{\prime}\right) / t=O(1 / t)$ so expanding in $t$ will fail for terms involving $\mathbf{p}$. Thus we must perform the $\mathbf{p}$ integral explicitly. At fixed $\mathbf{r}$, we change variables from $\mathbf{p}$ to $\boldsymbol{\Pi}$ :

$$
\begin{aligned}
K_{n l} & =-i \int^{\prime} D[\mathbf{r}] e^{i \int d \tau^{\prime} \mathbf{A} \cdot \dot{\mathbf{r}}} \Xi \\
\Xi & \equiv \int D[\boldsymbol{\Pi}] \int d \tau \mathcal{W}(\mathbf{r}(\tau), \boldsymbol{\Pi}(\tau)) e^{i \int d \tau^{\prime}\left[\mathbf{\Pi} \cdot \mathbf{r}-\Pi^{2} / 2 m\right]} \\
& =\int d \tau W\left(\mathbf{r}(\tau), \frac{-i \delta}{\delta \dot{\mathbf{r}}(\tau)}\right) \int D[\boldsymbol{\Pi}] e^{i \int d \tau^{\prime}\left[\mathbf{\Pi} \cdot \dot{\mathbf{r}}-\Pi^{2} / 2 m\right]} \\
& =\mathcal{N} \int d \tau W\left(\mathbf{r}(\tau), \frac{-i \delta}{\delta \dot{\mathbf{r}}(\tau)}\right) e^{i m / 2 \int d \tau^{\prime} \dot{\mathbf{r}}^{2}}
\end{aligned}
$$

Above, we use a functional derivative to pull $\mathcal{W}$ out of the $\Pi$ integral. This allows us to perform the Gaussian $\Pi$ integral, which yields a normalization constant $\mathcal{N}$.

Using the definition of $\mathcal{W}$ from Eq. (4), we have

$$
W\left(\mathbf{r}, \frac{-i \delta}{\delta \dot{\mathbf{r}}}\right)=\int d \mathbf{s} V_{n l}\left(\mathbf{r}+\frac{\mathbf{s}}{2}, \mathbf{r}-\frac{\mathbf{s}}{2}\right) e^{-\mathbf{s} \cdot \frac{\delta}{\delta \mathbf{r}}}
$$

The exponentiated functional derivative translates $\dot{\mathbf{r}}$ :

$$
\begin{aligned}
K_{n l}= & -i \mathcal{N} \int d \tau \int d \mathbf{s} \int^{\prime} D[\mathbf{r}] V_{n l}\left(\mathbf{r}(\tau)+\frac{\mathbf{s}}{2}, \mathbf{r}(\tau)-\frac{\mathbf{s}}{2}\right) \times \\
& e^{i \int d \tau^{\prime}\left(m\left[\dot{\mathbf{r}}-\mathbf{s} \delta\left(\tau^{\prime}-\tau\right)\right]^{2} / 2+\mathbf{A} \cdot \dot{\mathbf{r}}\right)}
\end{aligned}
$$

Note that changing the order of the integrals does not change the final result. We now change variables, at fixed $\mathbf{s}$ and $\tau$, from $\mathbf{r}(\tau)$ to $\mathbf{f}(\tau)$ as follows:

$$
\mathbf{r}\left(\tau^{\prime}\right)=\mathbf{f}\left(\tau^{\prime}\right)+\left[\mathbf{r}^{\prime}+\mathbf{s} \theta\left(\tau^{\prime}-\tau\right)+\left(\mathbf{r}-\mathbf{r}^{\prime}-\mathbf{s}\right) \tau^{\prime} / t\right] .
$$

where $d \theta(x) / d x=\delta(x)$. This choice follows from the action of $V_{n l}$ in Eq. (14): $V_{n l}$ causes a transition from $\mathbf{r}(\tau)-\mathbf{s} / 2$ to $\mathbf{r}(\tau)+\mathbf{s} / 2$ at time $\tau$. We parameterize the paths as fluctuations $\mathbf{f}$ about a straight-line path with a discontinuity of size $\mathbf{s}$ at time $\tau$ (term in brackets). With this choice, $\mathbf{f}$ obeys the conditions $\mathbf{f}(0)=\mathbf{f}(t)=0$. 
Upon substituting $\mathbf{f}$, we have

$$
\begin{aligned}
K_{n l}= & -i \mathcal{N} \int d \tau \int d \mathbf{s} \int D[\mathbf{f}] V_{n l}\left(\mathbf{r}(\tau)+\frac{\mathbf{s}}{2}, \mathbf{r}(\tau)-\frac{\mathbf{s}}{2}\right) \times \\
& e^{i m / 2\left(\mathbf{r}-\mathbf{r}^{\prime}-\mathbf{s}\right)^{2} / t+i \int d \tau^{\prime}\left(m \dot{\mathbf{f}}^{2} / 2+\mathbf{A} \cdot \dot{\mathbf{r}}\right)}
\end{aligned}
$$

The conditions on $\mathbf{f}$ permit the Fourier expansion $\mathbf{f}\left(\tau^{\prime}\right)=\sum_{n} \mathbf{f}_{n} \sin \left(\pi n \tau^{\prime} / t\right)$. The kinetic integral is $\int_{0}^{t} d \tau^{\prime} \dot{\mathbf{f}}^{2}=\pi^{2} / 2 \sum_{n} n^{2} \mathbf{f}_{n}^{2} / t$. We now note that for any analytic function $g(x), \int d x g(x) e^{i x^{2} / t}=\sqrt{i \pi t}[g(0)+$ $O\left(t^{2}\right)$ ], so that within an integral we may set $e^{i x^{2} / t}=\sqrt{i \pi t}\left[\delta(x)+O\left(t^{2}\right)\right]$. Since $K_{n l}$ is already $O(t)$ and we ignore terms of $O\left(t^{2}\right)$, we replace $e^{i x^{2} / t}$ by $\sqrt{i \pi t} \delta(x)$ everywhere in the above integral: this applies to the $\mathbf{f}_{n}^{2} / t$ as well as to the $\left(\mathbf{r}-\mathbf{r}^{\prime}-\mathbf{s}\right)^{2} / t$ terms. Physically, fluctuations in $\mathbf{f}$ and $\mathbf{s}$ contribute a wildly oscillating large phase as $t \rightarrow 0$ so only the stationary phase contribution at $\mathbf{f}=0$ and $\mathbf{s}=\mathbf{r}-\mathbf{r}^{\prime}$ remains when $t \rightarrow 0$.

Therefore, we set $\mathbf{f}=0, \mathbf{s}=\mathbf{r}-\mathbf{r}^{\prime}$, absorb all constants from the integrations into $\mathcal{N}^{\prime}$, and arrive at

$$
\begin{aligned}
K_{n l}= & -i \mathcal{N}^{\prime} \int d \tau e^{i \int d \tau^{\prime} \mathbf{A} \cdot \dot{\mathbf{x}}} \times \\
& V_{n l}\left(\mathbf{x}(\tau, \tau)+\frac{\mathbf{r}-\mathbf{r}^{\prime}}{2}, \mathbf{x}(\tau, \tau)-\frac{\mathbf{r}-\mathbf{r}^{\prime}}{2}\right), \\
\mathbf{x}\left(\tau, \tau^{\prime}\right) \equiv & \left.\mathbf{r}\left(\tau^{\prime}\right)\right|_{\mathbf{f}=0, \mathbf{s}=\mathbf{r}-\mathbf{r}^{\prime}}=\mathbf{r}^{\prime}+\left(\mathbf{r}-\mathbf{r}^{\prime}\right) \theta\left(\tau^{\prime}-\tau\right) .
\end{aligned}
$$

Since we defined $d \theta(x) / d x \equiv \delta(x)$ and $\delta(-x)=\delta(x)$, we have $\theta(0)=1 / 2$ so $\mathbf{x}(\tau, \tau)=\mathbf{r}^{\prime}+\left(\mathbf{r}-\mathbf{r}^{\prime}\right) / 2$.

The final hurdle involves evaluating $\int d \tau^{\prime} \mathbf{A} \cdot \dot{\mathbf{x}}$ in Eq. (16). We replace the $\delta(x)$ appearing in $\dot{\mathbf{x}}$ by any smooth, non-negative function $g(x)$ of compact support about $x=0$ with width much smaller than $t$ and unit integral. We then replace $\theta(x)$ by $G(x)$ where $d G(x) / d x=g(x)$, so $G$ increases monotonically from 0 to 1 in a width much smaller than $t$. The integral is then

$$
\int_{0}^{t} d \tau^{\prime} \mathbf{A}\left(\mathbf{x}\left(\tau, \tau^{\prime}\right), \tau^{\prime}\right) \cdot \dot{\mathbf{x}}\left(\tau, \tau^{\prime}\right)=\int_{\mathbf{r}^{\prime}}^{\mathbf{r}} \mathbf{A}(\mathbf{x}, t) \cdot d \mathbf{x}+O\left(t^{2}\right)
$$

We evaluate $\mathbf{A}$ at $t$ since any explicit time dependence of $\mathbf{A}$ changes $K_{n l}$ by $O\left(t^{2}\right)$, which is ignored. The integral is then just along a straight line from $\mathbf{r}^{\prime}$ to $\mathbf{r}$.

After replacing the $\tau$ integral by $t$ in Eq. (16), our nonlocal propagator is $K_{n l}=$ $-i t \mathcal{N}^{\prime} V_{n l}\left(\mathbf{r}, \mathbf{r}^{\prime}\right) \exp \left(i \int_{\mathbf{r}^{\prime}}^{\mathbf{r}} \mathbf{A} \cdot d \mathbf{x}\right)+O\left(t^{2}\right)$. Recovery of the correct propagator for $\mathbf{A}=0$ requires $\mathcal{N}^{\prime}=1$. Combining $K_{n l}$ with $K_{l o c}$ (Eq. (10)), we have as our central result the following gauge-invariant quantum Hamiltonian

$$
\begin{aligned}
\hat{H}_{\mathbf{A}} & =(\hat{\mathbf{p}}-q \hat{\mathbf{A}} / c)^{2} / 2 m+\hat{U}+q \hat{\phi}+\hat{V}_{n l}^{\mathbf{A}}, \\
\left\langle\mathbf{r}\left|\hat{V}_{n l}^{\mathbf{A}}\right| \mathbf{r}^{\prime}\right\rangle & =V_{n l}\left(\mathbf{r}, \mathbf{r}^{\prime}\right) e^{\frac{i q}{\hbar c} \int_{\mathbf{r}^{\prime}}^{\mathbf{r}} \mathbf{A}(\mathbf{x}, t) \cdot d \mathbf{x}} \text { (straight-line). }
\end{aligned}
$$

In practice, we often use perturbation theory to compute response functions: we set $\hat{H}_{A}=\hat{H}_{0}+\hat{H}^{i n t}$ and work to a desired order in the interaction $\hat{H}^{\text {int }}$. We expand the above result to all orders in $\mathbf{A}$ :

$$
\begin{gathered}
\hat{H}_{\mathbf{A}}=\hat{H}_{0}+q \hat{\phi}-\frac{q(\hat{\mathbf{p}} \cdot \hat{\mathbf{A}}+\hat{\mathbf{A}} \cdot \hat{\mathbf{p}})}{2 m c}+\frac{q^{2} \hat{\mathbf{A}}^{2}}{2 m c^{2}}+\hat{V}_{n l}^{i n t}, \\
\left\langle\mathbf{r}\left|\hat{V}_{n l}^{i n t}\right| \mathbf{r}^{\prime}\right\rangle=V_{n l}\left(\mathbf{r}, \mathbf{r}^{\prime}\right) \sum_{k=1}^{\infty} \frac{1}{k !}\left(\frac{i q}{\hbar c} \int_{\mathbf{r}^{\prime}}^{\mathbf{r}} \mathbf{A} \cdot d \mathbf{x}\right)^{k} .
\end{gathered}
$$

In relation to previous work, a coupling similar to Eq. (18) is given in Ref. [3] but only after the straight line path is assumed with no justification. Refs. [4] and 10 both find a result equivalent to the special case of Eq. (18) in the limit of spatially constant A. In Ref. [5], the linear term $(k=1)$ of Eq. 19) is derived correctly, but attempts at finding a general expression for arbitrary $\mathbf{A}$ are stymied by operator ordering problems. 


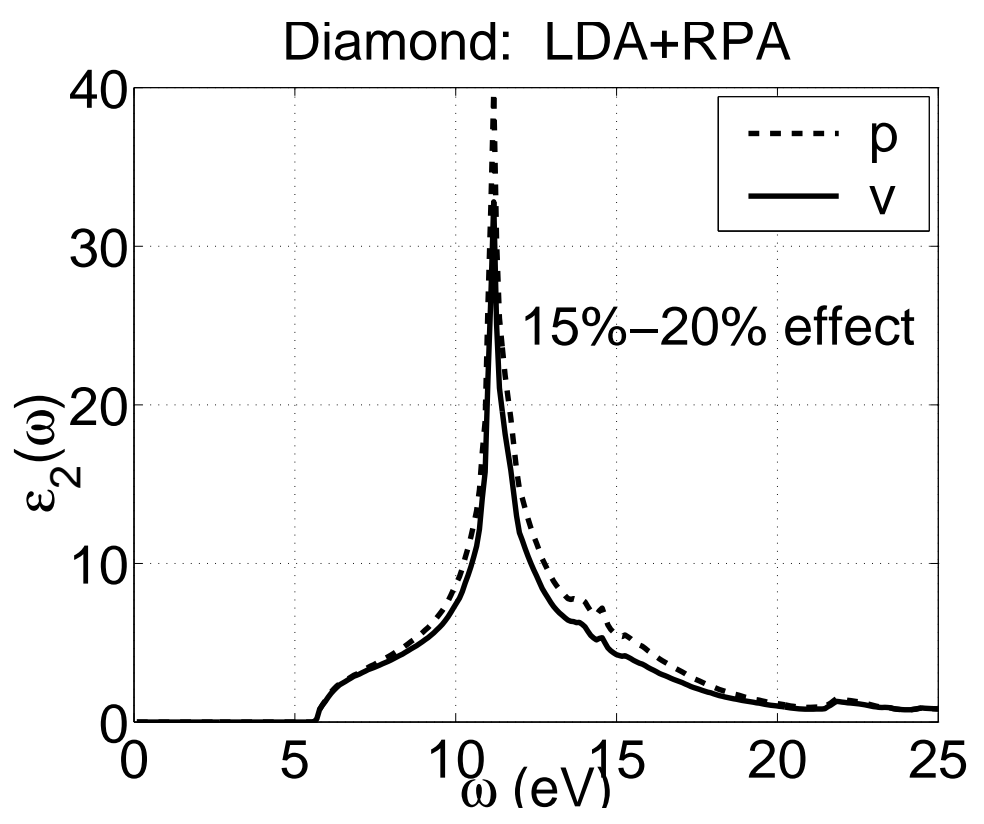

Figure 1: Absorption spectrum for diamond: $\varepsilon_{2}(\omega)$ vs. $\omega$ in eV. The dashed curve uses the momentum operator, the solid curve uses the velocity operator.

We now apply our result to the case of linear optical response where the wavelength of $\mathbf{A}$ is much greater than atomic dimensions and we set $\mathbf{A}$ constant over the nonlocal range of $V_{n l}$. For the Coulomb gauge $\nabla \cdot \mathbf{A}=\phi=0$, the linear coupling is $(k=1$ term of Eq. (19) $)$

$$
\hat{H}_{1}^{i n t}=-\hat{\mathbf{A}}(t) \cdot q \hat{\mathbf{v}} / c \text { where } \hat{\mathbf{v}} \equiv-i\left[\hat{\mathbf{r}}, \hat{H}_{0}\right] / \hbar .
$$

Here, $\hat{\mathbf{v}}=d \hat{\mathbf{r}} / d t$ is the velocity operator. This result justifies the intuition that $\mathbf{A}$ couples to the current $\mathbf{j}=q \mathbf{v}$. For a local system $\mathbf{v}=\mathbf{p} / m$ as expected. The difference between velocity and momentum has been stressed when computing optical response [6, 8, 10, 15]. However, those works assume the long wavelength limit or couple only to longitudinal fields. Our result is a direct demonstration that in the Coulomb gauge, the coupling of electrons to long waves is via the velocity operator. To show the significance of nonlocality, we perform $a b$ initio pseudopotential density functional calculations [16]. Figure 1 shows the RPA absorption spectrum, $\varepsilon_{2}(\omega)$ with a $0.2 \mathrm{eV}$ Lorentzian broadening, where either the momentum or velocity operator is employed. The effect of nonlocality is $15 \%$ to $20 \%$, non negligible when aiming for quantitative comparison to experiment.

Next, we study electrons in a uniform magnetic field. Magnetic fields provide a novel application of our result since A must vary spatially so that the usual long wavelength approximation is invalid. We calculate the magnetic susceptibility $\chi=-\partial^{2} E / \partial^{2} B$ of atomic carbon and neon. We choose $\mathbf{A}=-B(0,0, x+a)$ : the gauge center is offset from the ionic nucleus to simulate the realistic case when many atoms are present. We choose $a=4$ a.u. as a reasonable value. We use $s$ and $p$ Kleinmann-Bylander nonlocal projectors [16] ( $p$ local) and compute $\chi$ following ref. [7]. Calculating $\chi$ requires the linear and quadratic couplings, both of which have nonlocal terms. For linear coupling, using $\hat{\mathbf{v}}$ instead of $\hat{\mathbf{p}} / m$ has been advocated [7], whereas the quadratic nonlocal term of Eq. (19) is truly novel. Table 1 shows results for $\chi$ when using various possible couplings: (1) the traditional local coupling $(\hat{\mathbf{p}}-\hat{\mathbf{A}})^{2} / 2 m ;(2)$ the linear coupling is from Eq. (19) but the quadratic coupling is the local $\hat{\mathbf{A}}^{2} / 2 m$; (3) both linear and quadratic couplings are from Eq. (19); (4) all-electron results excluding the contribution of the core $1 s$ states. Method (1) is qualitatively incorrect as it is not gauge-invariant. Using the correct linear coupling (method 2) greatly improves the quality of the results. However, the inclusion of our novel nonlocal quadratic terms (method 3) yields $\chi$ in excellent quantitative agreement with the desired all-electron results.

We emphasize that Eq. (18) gives the coupling to any order in A. This allows, for the first time, for direct 


\begin{tabular}{c||c|c|c|c|c}
$-\chi$ & 1 & 2 & 3 & all-elec. & $\operatorname{expt}[7]$ \\
\hline \hline $\mathrm{C}$ & -40.1 & 16.9 & 12.80 & 12.85 & - \\
\hline $\mathrm{Ne}$ & -62.4 & 6.15 & 7.76 & 7.75 & 7.2
\end{tabular}

Table 1: Negative of valence atomic magnetic suceptibility $-\chi$ in $\mathrm{cm}^{3} /$ mole. See text for details.

and systematic calculation of high-order nonlinear responses without resort to longitudinal-only couplings [8] or to the long wave limit for the transverse case [9, 10, 17].

Finally, our result bears directly on systems with generic nonlocal hopping terms, e.g. tight-binding systems, where electrons hop from a localized state $\beta$ on site $\mathbf{R}^{\prime}$ to a state $\alpha$ at $\mathbf{R}$ with amplitude $t_{\alpha \beta}\left(\mathbf{R}, \mathbf{R}^{\prime}\right)$. Our result shows that, in the extreme tight-binding limit, the EM coupling modifies the amplitude to become

$t_{\alpha \beta}\left(\mathbf{R}, \mathbf{R}^{\prime}\right) \exp \left(\frac{i q}{\hbar c} \int_{\mathbf{R}^{\prime}}^{\mathbf{R}} \mathbf{A} \cdot d \mathbf{x}\right)$. This justifies the straight-line integral used in the Peierls substitution and does not suffer from path ambiguity 11 .

This work was supported by NSF grant \#DMR-0087088 and by the Office of Energy Research, Office of Basic Energy Sciences, Materials Science Division of the U.S. Department of Energy contract \#DEAC03-76SF00098. We thank Kevin Mitchell and Andrew Charman for illuminating the importance of Weyl transformations. Young-Gui Yoon provided helpful criticism of the ideas leading to this work. Computer time was provided by the DOE at the Lawrence Berkeley National Laboratory's NERSC center.

\section{References}

[1] See, e.g., articles in Quantum Theory of Real Materials, J. R. Chelikowsky and S. G. Louie, eds. (Kulwer Academic Publishers, Norwell MA (1996)).

[2] J. M. Luttinger, Phys. Rev. 84, 814 (1951); J. M. Luttinger and W. Kohn, Phys. Rev. 97, 869 (1955); W. Kohn, Phys. Rev. 115, 1460 (1959).

[3] A. M. Kolorev, Sov. J. Nuc. Phys. 6, 256 (1968).

[4] A. F. Starace, Phys. Rev. A 3, 1242 (1971).

[5] H. Zhong, Z. H. Levine, D. C. Allan, and J. W. Wilkins, Phys. Rev. B 48, 1384 (1993).

[6] S. Baroni and R. Resta, Phys. Rev. B 33, 7017 (1986); M. S. Hybertsen and S. G. Louie, Phys. Rev. B 35, 5602 (1987).

[7] F. Mauri and S. G. Louie, Phys. Rev. Lett. 76, 4246 (1996).

[8] Z. H. Levine and D. C. Allan, Phys. Rev. Lett. 63, 1719 (1989); Phys. Rev. B 42, 3567 (1990); 43, 4187 (1991); 4412781 (1991).

[9] A. Dal Corso, F. Mauri, A. Rubio, Phys. Rev. B 53, 15638 (1996).

[10] R. Girlanda, A. Quattropani and P. Schwendimann, Phys. Rev. B 24, 2009 (1981); A. Quattropani and R. Girlanda, Solid State Commun. 38, 1041 (1981); E. Piparo and R. Girlanda, Il Nuovo Cimento 14D, 679 (1992); R. Del Sole and R. Girlanda, Phys. Rev. B 48, 11789 (1993).

[11] M. Graf and P. Vogl, Phys Rev. B 51, 4940 (1995) and references therein.

[12] H. Weyl, Z. Phys. 46, 1 (1927); E. P. Wigner, Phys. Rev. 40, 749 (1932); M. Müller, J. Phys. A 32, 1035 (1999) and references therein.

[13] J. W. Negele and H. Orland, Quantum Many-particle Systems, Perseus Books, Reading (1998).

[14] A. M. Zagoskin, Quantum Theory of Many-Body Systems, Springer-Verlag, New York (1998). 
[15] B. Adolph, V. I. Gavrilenko, K. Tenelsen, F. Bechstedt, and R. Del Sole, Phys. Rev. B 53, 9797 (1996).

[16] M. C. Payne, M. P. Teter, D. C. Allan, T. A. Arias, and J. D. Joannopoulos, Rev. Mod. Phys. 64, 1045 (1992), and references therein.

[17] G. F. Bertsch, J.-I. Iwata, A. Rubio, and K. Yabana, Phys. Rev. B 62, 7998 (2000). 